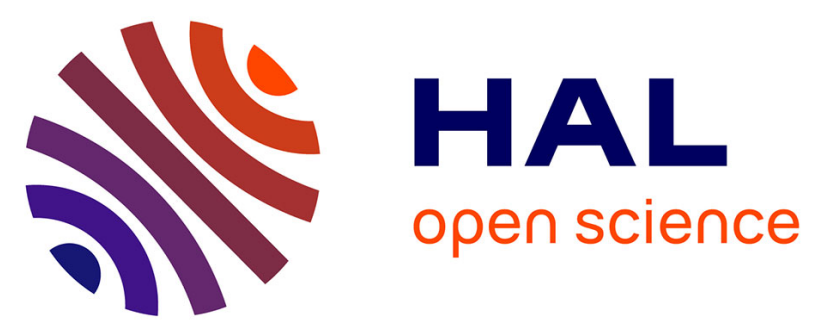

\title{
Sensor-based control with digital maps association for global navigation: a real application for autonomous vehicles
}

\author{
Danilo Alves de Lima, Alessandro Corrêa Victorino
}

\section{To cite this version:}

Danilo Alves de Lima, Alessandro Corrêa Victorino. Sensor-based control with digital maps association for global navigation: a real application for autonomous vehicles. 18th IEEE International Conference on Intelligent Transportation Systems (ITSC 2015), Sep 2015, Las Palmas de Gran Canaria, Spain. pp.1791-1796, 10.1109/ITSC.2015.291 . hal-01305752

HAL Id: hal-01305752

https://hal.science/hal-01305752

Submitted on 21 Apr 2016

HAL is a multi-disciplinary open access archive for the deposit and dissemination of scientific research documents, whether they are published or not. The documents may come from teaching and research institutions in France or abroad, or from public or private research centers.
L'archive ouverte pluridisciplinaire HAL, est destinée au dépôt et à la diffusion de documents scientifiques de niveau recherche, publiés ou non, émanant des établissements d'enseignement et de recherche français ou étrangers, des laboratoires publics ou privés. 


\title{
Sensor-based control with digital maps association for global navigation: a real application for autonomous vehicles
}

\author{
Danilo Alves de Lima and Alessandro Corrêa Victorino
}

\begin{abstract}
This paper presents a sensor-based control strategy applied in the global navigation of autonomous vehicles in urban environments. Typically, sensor-based control performs local navigation tasks regarding some features perceived from the environment. However, when there is more than one possibility to go, like in road intersection, the vehicle control fails to accomplish its global navigation. In order to solve this problem, we propose the vehicle global navigation based on a topological representation of the environment using only digital maps and low cost sensors. The approach was developed for two main tasks: road following and road intersection maneuvers. The final solution was completely implemented in a real autonomous car and tested in a challenge circuit, showing the viability of our solution.
\end{abstract}

Index Terms-Sensor-based navigation, Global navigation management, Digital maps.

\section{INTRODUCTION}

The complete navigation of self-driving cars is the ability to go from an initial position $\left(q_{\text {init }}\right)$ to a final destination $\left(q_{\text {goal }}\right)$ in the world, considering that these points are not connected in the robot's workspace. To achieve that, motion planning is fundamental to define a feasible path connecting these points. In this context, several approaches were proposed in the past years for nonholonomic car-like robots motion planning [1], [2], defined in the car's configuration space. However, these strategies normally presents a high computational cost associated with the size of the urban environments, mainly if it is dynamic.

To deal with these problems, it is common to consider the vehicle as a punctual holonomic robot during the path planning and simplify the environment in geometric forms and segments. These elements edges define the points where the robot must pass (waypoints). Waypoints can be seen as a simplification of the entire robot path, recovered by connecting each consecutive waypoint. The navigation based on waypoints was applied by several participants in the DARPA Grand Challenge of 2005 for off-road autonomous vehicles [3], provided in the route definition data format (RDDF). Although the waypoints were able to guide the vehicles, they were previously defined by the organizers of the challenge with high precision sensors. In addition, the robots have required accurate localization systems while performing the path following tasks, impracticable for commercial cars.

In urban environments, one way to define waypoints is using road network maps, also known as digital maps. Digital

\footnotetext{
The authors are with Heudiasyc UMR CNRS 7253 Université de Technologie de Compiègne. Danilo Alves Lima holds a Ph.D scholarship from Picardie region. Contact authors danilo.alves-de-limalhds.utc.fr
}

maps provide rich information about streets, tracks, railways, waterways, points of interest, also detailing the number of lanes and speed limits. There are many approaches using digital maps to improve the global navigation problem, like enhancing the vehicle localization [4] and the difficulties of autonomous vehicles navigation as a whole [5]. Based on digital maps, the DARPA Urban Challenge [6] organizers provided the road network definition file (RNDF) to the participants. However, the RNDF itself was inaccurate and could add further errors if the vehicle blindly follow the road using that information. Thus, the participants locally planned paths between the checkpoints, defining waypoints to guide the robot navigation and correct the robot lateral position by means of a high precision localization system and the RNDF information. Recently, Ziegler et al. [7] performed the autonomous navigation in urban environments using a prerecorded reference trajectory (waypoints) for the vehicle. They use a DGPS-aided inertial navigation system during the recording process, adding more environment information to the digital map (like lanes number, speed limits, stop signs, etc.) in a post-processing step. This post processing was performed in a user-generated street map interface, named as OpenStreetMap (OSM) [8]. Thus, during the real car navigation, a map based localization was performed with a less costly system.

Although all these previous methods were able to guide globally the robot, locally they were highly dependent on the localization system. Victorino et al. [9] proposed a new navigation approach for indoor environments with a topological representation of the robot path. In this case, each corridor was connected by nodes representing the relations of accessibility in the environment (doors and crossing corridors). Similarly, in urban environments, corridors are roads, without any-other way to go, and nodes are points where some action must be taken (like turn to a next road in the intersection or stop in a traffic light). In our previous work [10], the nodes were called by critical points (CPs), where, in comparison to waypoints, they are sparsely defined and do not represent the path's form if connected. Thus, the robot localization is not required during the road following navigation (due to the absence of waypoints), and some sensor-based approach is enough to guide the vehicle to the next $\mathrm{CP}$. At the $\mathrm{CP}$, the vehicle localization can be performed by low cost systems, which can be less dependent on GPS sensors [11].

In this work, the goal is to enable the global navigation of a car-like robot using local navigation techniques and low cost sensors, mainly when there is more than one possibility 


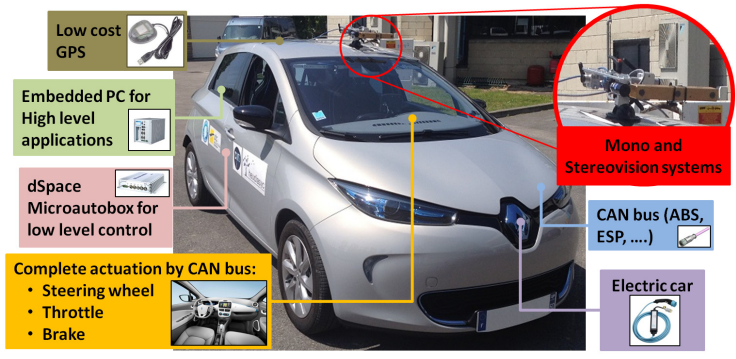

Fig. 1. Fully actuated electric car APACHE with some available resources.

to go. For that, it is fundamental to provide information associated to the CPs, as their connections and approximated geometric shape. Thus, digital maps (like the OSM) are important tools for this purpose. Differently from our previous work [10], the robot navigation at intersections will be generalized and validated in a real car-like robot. In addition, the final robot control will be performed by a sensor-based strategy, using the benefits of the local navigation controller $\mathrm{VS} \mathrm{IDWA}^{1}$ [12].

This work was structured as follows: the Section II shortly describes the real car-like robot used in the experiments and some available resources; the routing table with the topological representation of the robot path is described in the Section III; Section IV presents the sensor-based approach for the global navigation, with the management of the routing table information; some navigation experiments in our real car are presented in the Section V; and the final considerations and future works are listed in the Section VI.

\section{SySTEM DESIGN}

The experimental car is an electric Renault Zoe, hereby appointed APACHE and presented in the Figure 1. It was modified for computational actuation of throttle, brake, and steering, receiving commands over the CAN bus. Although these are important modifications, the vehicle still preserving the original human driver conduction capabilities, allowing e.g. a cooperative working mode between the human driver and the computer system. Besides the low level control (steering, throttle, and brake), several proprioceptive information can be retrieved from the CAN bus. Some of them are illustrated in the Figure 1, like the ABS and odometry from the wheels, the linear and steering velocities, the steering angle, etc.

This vehicle was equipped with two PointGrey cameras (Flea3 and BumblebeeXB3) for environment perception and a GPS for global localization (Haicom HI-204E), chosen as a low cost alternative for exteroceptive sensors. The monocular camera (Flea3) has a focal length of $1.8 \mathrm{~mm}$ and large field of view (FOV) of $\simeq 140^{\circ}$ to detect the road lane center features. The stereovision camera (BumblebeeXB3) has a focal length of $6.0 \mathrm{~mm}$ and $\mathrm{FOV} \simeq 43^{\circ}$, which allow the vehicle to detect road and obstacles up to $80 \mathrm{~m}$ away from it. Both cameras were installed on a rigid structure on the car roof only for

\footnotetext{
${ }^{1} \mathrm{VS}+\mathrm{IDWA}$ is an acronym for a hybrid controller composed by Visual
} Servoing (VS) and Imaged-based Dynamic Window Approach (IDWA). validation purposes. The GPS works at $1 \mathrm{~Hz}$ and produce position errors in a radius of $\approx 5 \mathrm{~m}$.

To allow the implementation and execution of this navigation strategy, some embedded computers for data processing and vehicle control were installed in the APACHE (Figure 1). These computers implement:

- The vehicle localization based on GPS and CAN data fusion, by an extended Kalman Filter (EKF), with a final error of $\pm 5 \mathrm{~m}$ at $10 \mathrm{~Hz}$;

- The low level velocity control, providing setpoints for the throttle, brake, and steering actuators;

- The environment perception, with the road/obstacles detection and their representation in a local occupancy grid;

- The local navigation control, implementing the VS+IDWA approach [12], used as a sensor-based control of our global navigation management; and

- The global navigation management, presented in the Section IV, to address the vehicle state in the routing table and provide the right parameters to the local navigation control.

\section{Global Route Planning}

To validate the present navigation approach, roads were considered as corridors and their intersections as critical points (CPs), similarly to [10]. Based on digital maps, provided by the OSM, a routing table was created by structuring the global navigation from $q_{\text {init }}$ to $q_{\text {goal }}$ after a path planning. Differently from [10], we divided this table in two parts, where the first one is related to the road (way) description after the $\mathrm{CP}$, and the second part represents the $\mathrm{CP}$ geometry with the angle and direction of every intersecting road. The first part has a constant size, once the possible road parameters are always the same, and the second one has the size depending on the number of roads intersecting the CP. The columns are:

- Node: with the path's CPs ordered from $q_{\text {init }}$ to $q_{\text {goal }}$;

- Lat and Long: latitude and longitude coordinates of the CPs in degrees;

- $\mathbf{V}_{\text {max }}$ : maximum speed limit of the $\operatorname{road}($ in $\mathrm{km} / \mathrm{h}$ );

- $\mathbf{R}_{\text {lanes }}$ : number of available lanes in the road (even if it is a two way road);

- $\mathbf{R}_{\text {way }}$ : road classification in one way (1) or not (2);

- $\mathbf{R}_{\mathbf{a}}$ : CP definition in roundabout point or not;

- $\mathbf{N}_{\text {roads }}$ : number of roads at each $\mathrm{CP}$;

- $\boldsymbol{\Theta}_{1 . . \mathbf{N}_{\text {roads }}}$ and $\mathbf{D}_{1 . . \mathbf{N}_{\text {roads }}}$ : sets of angle and direction for each road at the $\mathrm{CP}$, ordered counterclockwise. $\Theta_{1 . . N_{\text {roads }}}$ are formed angles between the current road and the next one in the intersection. $D_{1 . . N_{\text {roads }}}$ indicate if the road is the path to follow(4), a wrong way (arrival) road (3), a right way (departure) $\operatorname{road}(2)$, an arrival/departure $\operatorname{road}(1)$, or if it is the goal point $(0)$.

To illustrate these parameters, the Table I presents the routing table for the condition shown in the Figure 2. A complete routing table based on the OSM data will be presented in the experimental results of this work (Section V). A global 
TABLE I

ROUTING TABLE FOR THE PATH REPRESENTED IN THE FIGURE 2.

\begin{tabular}{|c|c|c|c|c|c|c|c|c|c|c|c|}
\hline \multicolumn{9}{|c|}{ CP description } & \multicolumn{4}{c|}{ CP geometry } \\
\hline Node & Lat & Lon & $\mathbf{V}_{\text {max }}$ & $\mathbf{R}_{\text {lanes }}$ & $\mathbf{R}_{\text {way }}$ & $\mathbf{R}_{\mathbf{a}}$ & $\mathbf{N}_{\text {roads }}$ & $\boldsymbol{\Theta}_{\mathbf{1}}$ & $\mathbf{D}_{\mathbf{1}}$ & $\boldsymbol{\Theta}_{\mathbf{2}}$ & $\mathbf{D}_{\mathbf{2}}$ \\
\hline$q_{\text {init }}$ & 49.392 & 2.798 & 30 & 1 & 1 & 0 & 1 & 180 & 4 & - & - \\
\hline 1 & 49.400 & 2.800 & 50 & 2 & 2 & 0 & 2 & 110 & 4 & 290 & 1 \\
\hline$\vdots$ & $\vdots$ & $\vdots$ & $\vdots$ & $\vdots$ & $\vdots$ & $\vdots$ & $\vdots$ & $\vdots$ & $\vdots$ & $\vdots$ & $\vdots$ \\
\hline
\end{tabular}

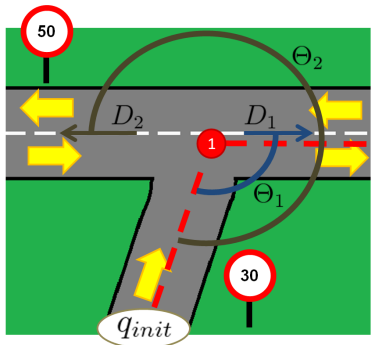

Fig. 2. Example of a road intersection to illustrate the routing table definitions, where the yellow arrows indicates the traffic way.

navigation, based on the routing table, can be performed by following the directions indicated as path to follow (4) at each CP. The management of this table during a sensor-based navigation is presented next.

\section{Sensor-Based Global Navigation}

For the global navigation problem of guiding the autonomous car from $q_{\text {init }}$ to $q_{\text {goal }}$, in urban environments, two local tasks were considered: road following (corridors) and road intersection maneuvers (at CPs). In this context, we proposed the association of a sensor-based controller for local navigation in order to accomplish both tasks, using the environment perception data (from exteroceptive sensors) and the path information from the routing table (previously defined in the Section III). This section presents the navigation management to switch between these two tasks, the local navigation control principle, and its application in the road following and road intersection maneuvers tasks.

\section{A. Navigation management}

The role of the navigation management is to determine which local task must be performed (road following or road intersection maneuver) during the car-like robot movement from $q_{\text {init }}$ to $q_{\text {goal }}$, with relation to the routing table for its planned path. These tasks can be distinguished by defining how near/far from a CP is the robot. For that, we performed the APACHE movement in a track with CPs defined in all road intersections, presented in the Figure 6. The resulting trajectory, provided by its localization system, always intersects the black circles centered in the CPs. The circles consider the maximum robot localization and OSM errors, resulting in 10 meters of radius. Thus, the vehicle is near to a $\mathrm{CP}$ when the distance between them is lower than 10 meters and faraway otherwise. Approaching a $\mathrm{CP}$, the maximum vehicle speed can be reduced to improve the navigation

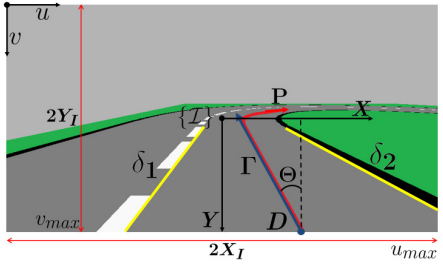

Fig. 3. Image frame $\{\mathcal{I}\}$ representation for the camera installed on the sagittal plane of the car's roof. The road lane center projection $P$ (in red) is related to the boundaries $\delta_{1}$ and $\delta_{2}$ (in yellow). Its tangent $\Gamma$ (in blue) at the point $D$ with angle offset $\Theta$ from $\Gamma$ to the axis $-Y$, defines the image features $X, Y$, and $\Theta$.

behavior and also apply some localization technique based on the local information [11]. After selecting the local task, the navigation management must define the image setpoint and the control parameters based on the robot perception system for the local navigation control.

\section{B. Local navigation control}

The sensor-based approach used for the local navigation control is a hybrid solution for road lane following named as VS+IDWA [12]. Using cameras as the main sensors, it combines a Visual Servoing (VS) technique as a deliberative control, for road lane following, and an Image-based Dynamic Window Approach (IDWA), for reactive obstacle avoidance. The focus of this controller is to guide the image features set $s=\left[\begin{array}{lll}X & Y & \Theta\end{array}\right]^{T}$ to the desired configuration $X=X^{*}, Y=Y^{*}$, and $\Theta=\Theta^{*}$, while avoiding obstacles. At the Figure 3, the features' set was extracted from a line formed by the tangent $\Gamma$ of the path $P$ at the point $D=(X, Y) . \Theta$ represents the angular offset from $\Gamma$ to the axis $-Y$ (positive counterclockwise). The path $P$ is defined by the road lane center between the boundaries $\delta_{1}$ and $\delta_{2}$.

In this work, the camera was installed on the car's sagittal plane and the desired features configuration represents the car in the center of the road lane, which means the line vertically in the center of the image frame. Thus, for the global navigation, the right image features must be provided to the VS+IDWA controller when performing the road following or the road intersection maneuver. Moreover, the environment information must be modified to avoid invalid movements and the robot speed limit adjusted during the navigation.

\section{Local navigation: road lane following}

Once the robot is on the road following state, the VS+IDWA controller [12] can be applied as it was conceived to accomplish the current local navigation task. In this case, 
the environment perception capabilities of our experimental car APACHE is responsible to detect and provide the correct image feature, as defined in the Figure 3 . With the previous knowledge about road speed limit $\left(V_{\max }\right)$, number of lanes $\left(R_{\text {lanes }}\right)$, and traffic way $\left(R_{\text {way }}\right)$, provided by the routing table, the speed setpoint can be adjusted and the lane detection algorithm optimized for each road. In addition, with the traffic way, the obstacle overtaking can be better controlled, avoiding, e.g., movements in discordance with the traffic laws.

\section{Local navigation: road intersection maneuvers}

Approaches for road following do not traditionally deal with road intersection maneuvers, requiring some intervention at these situations. With this problem in mind, we propose a complement for the VS+IDWA controller [12], generalizing the idea presented in [10]. The first part of this approach is described in the Algorithm 1, with the detection of the road intersection corners and the main steps illustrated in the Figure 4. It was inspired by the laser detection of tunnel intersections [13], where a similar laser reading in the local occupancy grid defines the obstacles position in polar coordinates $\left(r_{\text {obst }}, \theta_{\text {obst }}\right)$ in a maximum perceived distance $d_{\max }$ (see the pink dots in the Figure $4 \mathrm{~b}$ ). It also uses the car's width $C_{w i d t h}$, to eliminate false positives, and the routing table information of $N_{\text {roads }}$, with the number of roads at the intersection and their geometry (Figure 4c). These parameters define the path possibilities for the current robot state (Figure 4a), represented by the arrows (red and yellow colors) in the navigable area (black pixels) of the occupancy grid.

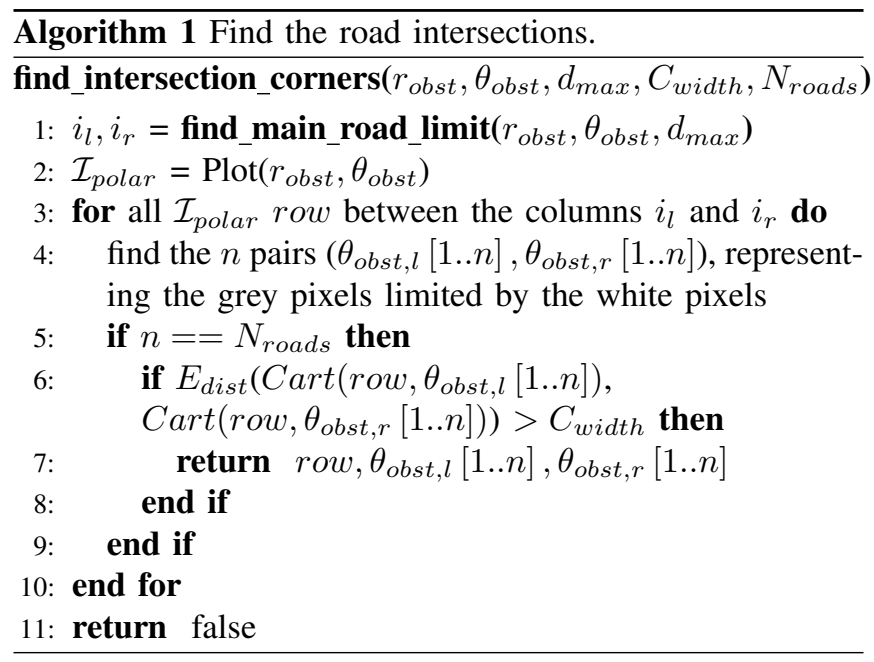

Following this algorithm, initially the main road limit is calculated by the Algorithm 2, which return $i_{l}$ and $i_{r}$. They represent the left and right $\theta_{\text {obst }}$ indexes with the smallest distance to the vehicle. These points reduce the search algorithm to the region defined between them. The final search is performed in the polar coordinates graphic $\mathcal{I}_{\text {polar }}$ (Figure $4 \mathrm{~d}$ ), with the radius measurements linked by the white line dividing the area among the vehicle (grey

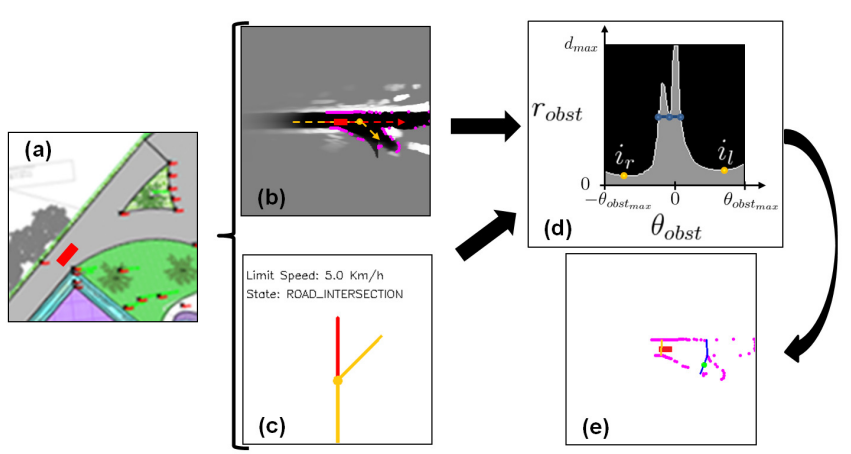

Fig. 4. Steps to find the road intersection corners for the car pose (a). Its respective local occupancy grid (b) and routing table state (c) illustrate the road structure at the intersection and the end of the navigable area given by pink dots with polar coordinates $\left(r_{\text {obst }}, \theta_{\text {obst }}\right)$. These polar coordinates were used to detect the main road limits and to generate the graph (d), which defines the road intersections corners (in blue). They are better visualized in the grid point of view in (e), with the main road in yellow, the intersections in blue, and the destination road center in green.

color) and faraway (black color). Thus, the algorithm search for the row discontinuities, which represent the white-grey and grey-white variations between $i_{l}$ and $i_{r}$. These points are the candidates for the road intersection corners and must be checked by the following two filters:

- $\mathbf{N}_{\text {roads }}$ : verify if the number of $n$ candidates is equal to the amount of expected roads at the intersection. Each candidate is equal to the pair formed by the whitegrey (row, $\left.\theta_{\text {obst }, l}[k]\right)$ and grey-white (row, $\theta_{\text {obst }, r}[k]$ ) variation, for $k=[1 . . n]$;

- $\mathbf{E}_{\text {dist }}$ : test if the Euclidean distance of each candidate, in Cartesian coordinates (Cart), is higher than the car's width $\left(C_{\text {width }}\right)$.

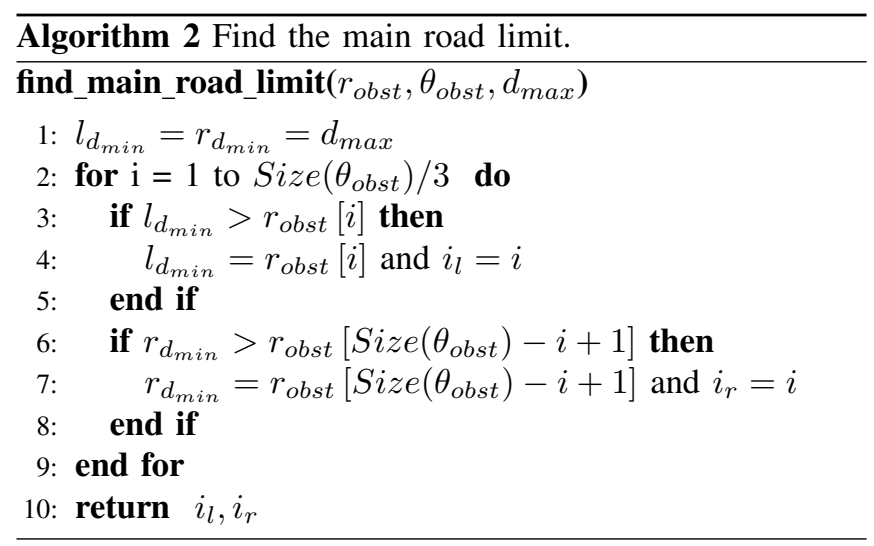

The valid candidates are illustrated by blue dots in the Figure $4 \mathrm{~d}$. The main road limits and the intersections found are represented in the grid point-of-view in the Figure 4e. By the intersection geometry, the wrong vehicle directions are obstructed with virtual obstacles (also known as geofencing) at the distance equal to row, which results in the Figure $5 \mathrm{a}$. Once the VS+IDWA requires an image features set for the control calculation, the line connecting the vehicle to the next valid road center (green point at the Figure 5a) was projected 
TABLE II

ROUTING TABLE FOR THE GLOBAL NAVIGATION MANAGEMENT EXPERIMENTS.

\begin{tabular}{|c|c|c|c|c|c|c|c|c|c|c|c|}
\hline \multicolumn{8}{|c|}{ CP description } & \multicolumn{4}{|c|}{ CP geometry } \\
\hline Node & Lat & Lon & $\mathbf{V}_{\max }$ & $\mathbf{R}_{\text {lanes }}$ & $\mathbf{R}_{\text {way }}$ & $\overline{\mathbf{R}_{\mathrm{a}}}$ & $\mathbf{N}_{\text {roads }}$ & $\Theta_{1}$ & $\mathrm{D}_{1}$ & $\Theta_{2}$ & $\mathrm{D}_{2}$ \\
\hline$q_{\text {init }}$ & 49.4021061 & 2.7948685 & 13 & 1 & 1 & 0 & 1 & 180 & 4 & - & - \\
\hline 1 & 49.4024539 & 2.7953365 & 7 & 1 & 2 & 0 & 2 & 135 & 4 & 180 & 3 \\
\hline 2 & 49.4024597 & 2.7955631 & 5 & 1 & 2 & 1 & 2 & 180 & 4 & 330 & 3 \\
\hline 3 & 49.4026169 & 2.7955953 & 7 & 1 & 1 & 1 & 2 & 180 & 4 & 210 & 2 \\
\hline 4 & 49.4024800 & 2.7953713 & 13 & 1 & 2 & 0 & 2 & 180 & 4 & 315 & 2 \\
\hline$q_{\text {goal }}$ & 49.4021061 & 2.7948685 & 13 & 1 & 0 & 0 & 1 & 180 & 0 & - & - \\
\hline
\end{tabular}

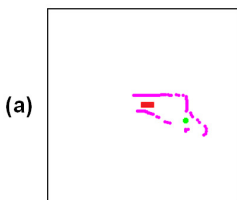

(b)

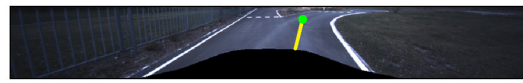

Fig. 5. Final $r_{o b s t}$ and $\theta_{o b s t}$ with the virtual obstacles defined by the method described in the Algorithm 1 (a) and the new image features (b), oriented to the center of the next road (green point).

in the image plane and defined as the new image features, as seen in the Figure 5b. For more details about the projection principle, see [12].

This approach is always executed when the vehicle is on the road intersection. Thus, the center of the destination road (green point) can be used as a condition to check if the robot has reached the destination road. If this point is closer enough to the front of the car, the road intersection maneuver was successfully performed and we can switch to the next local navigation method.

\section{EXPERIMENTAL RESULTS}

To validate the proposed global navigation management, it was completely implemented in the experimental car APACHE (see Section II), using its resources for environment perception and local navigation control. This vehicle is an important test bed for our navigation approach, but, due to the current modifications, it is still not allowed to run in the city roads. Thus, at the Innovation Center of the Université de Technologie de Compiègne was constructed a circuit called SEVILLE for autonomous navigation experiments. It presents some challenging scenarios, like tight road intersections, roundabouts, sharp turns, and up and downhills $\left(\approx 3.5^{\circ}\right)$, which was used during the validation of our navigation approach.

With the system running at $10 \mathrm{~Hz}$, a complete experiment was carried out to present the global navigation management capabilities during the road following and the road intersection maneuvers in full autonomous mode. The Table II describes the routing table in this situation using only the OSM data. Once this is a closed circuit, the path planning was not applied, requiring only the order of the CPs where the vehicle must pass. The localization tolerance for each $\mathrm{CP}$ was set to $10 \mathrm{~m}$, regarding the GPS and OSM errors.

The Figure 6 illustrates the global navigation movement performed by the APACHE for the CPs of Table II. It
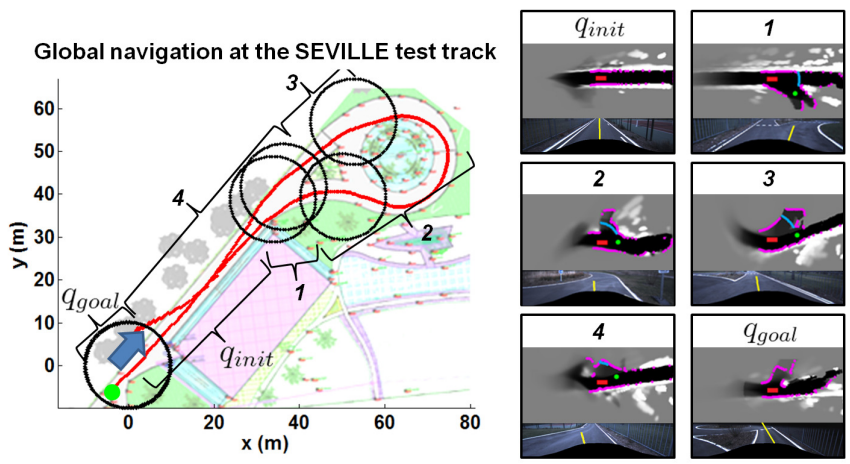

Fig. 6. Global navigation experiment for the routing table defined on Table II. The initial position is defined by the blue arrow and the destination by the green point in the map. The robot positions are illustrated in red and the localization tolerance around each CP are represented by the black circles. Some screenshots of the environment perception system illustrate the management during the road following and intersection maneuvers tasks.

also presents some screenshots from the environment perception system to illustrate the car navigation during the road following and intersection maneuvers tasks. In this figure, the results for the local navigation control based on the VS+IDWA [12] were confirmed, with the robot moving smoothly during the path, trying to bring the image features error to the zero condition (vertically in the center of the image), and avoiding the obstacles detected in the occupancy grid (pink dots for real obstacles and blue dots for virtual ones). Note that the localization system variations make path following applications using only GPS points unfeasible, but they are well fitted in the CPs tolerance radius.

On this previous movement, the global navigation management performs the speed limitation for each local road. The Figure 7 shows the linear velocity profile for the complete movement with the limitation performed for each routing table row. In this figure, the global management is evidenced during the $\mathrm{CP}$ transitions, where the road nominal speed (defined as 5,7 , and $13 \mathrm{~km} / \mathrm{h}$ ) is limited to $5 \mathrm{~km} / \mathrm{h}$ to allow safe intersection maneuvers. In order to mimic a human driver behavior and prepare the vehicle for the arrival of an intersection, we anticipate the speed limitation to $5 \mathrm{~m}$ before the CPs. This distance allows a smooth arrival in the intersection for the current speed limitations. However, for high speed applications, this distance must be related to the vehicle maximum deceleration for a smooth speed reduction.

In addition, the Figure 8 presents in detail one of these 


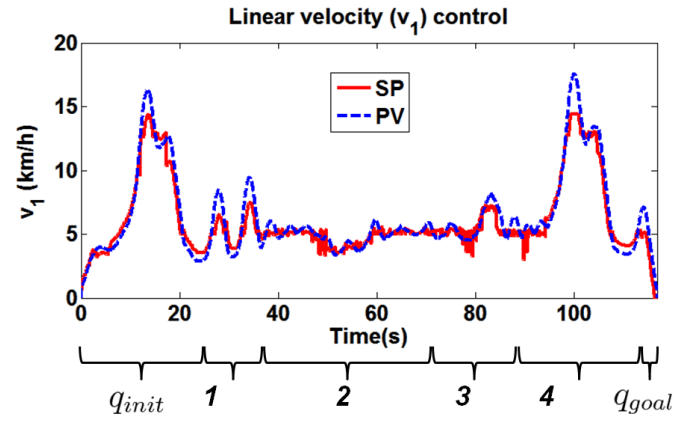

Fig. 7. Velocity profile for the global navigation experiment I of Figure 6.

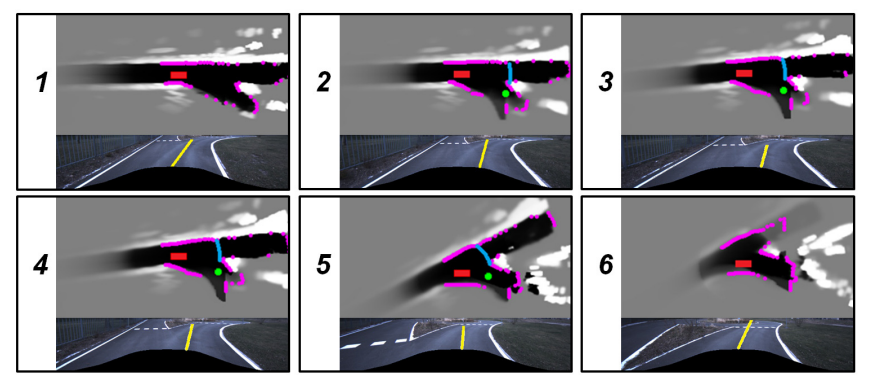

Fig. 8. Vehicle movement during a road intersection maneuver, emphasizing the virtual obstacles (blue dots) and the destination road center (green dot) used to define the new image feature in these conditions (yellow line).

maneuvers, with some sequential frames. In these frames it is possible to see the robustness of this approach during the movement, detecting the intersecting road with low variations as well as the next road center (green dot). Note that the roads geometry at the intersection do not matter on fact, but can be considered to enhance the detection capabilities. The most important element here is a good representation of the road area by the perception system. If the next road surface is not well detected for example, or it is not on the sensor's field of view, the vehicle will not be able to exit from the intersection situation.

\section{CONCLUSiOnS AND FUtURE WORKS}

This work presented a new sensor-based approach to deal with the global navigation problem of car-like robots in urban environments, using only low cost sensors and digital map data. We focused on two main tasks, road following and road intersection maneuvers, performed with our local navigation controller VS+IDWA. The final system integrated all the available resources of the experimental vehicle APACHE and successfully accomplished the two main tasks, following the image reference and avoiding obstacles, real or not. The results showed the viability of the solution, even in the presence of a reduced number of sensors for environment perception. Nevertheless, increasing the field of view and, consequently, the number of sensors used is fundamental to assure safe navigation in more situations. Future works will enhance the actual perception configuration for better intersection detection. Moreover, better localization techniques should be tested to avoid GPS problems in urban canyons when approaching an intersection. In addition, more road constraints must be considered, like road lanes, stop signs, the road priority, etc., to allow road following and intersection maneuvers closer to real situations.

\section{ACKNOWLEDGEMENT}

This work was carried out and funded in the framework of the Equipex ROBOTEX (Reference ANR-10-EQPX-4401). It was equally supported by the French Picardie project VERVE, French Government, through the program "Investments for the future" managed by the National Agency for Research, and the European Fund of Regional Development FEDER. The authors wish to thank the helpful assistance of Gérald Dherbomez, Giovani B. Vitor, Pierre Hudelaine, and Thierry Monglon during the experiments.

\section{REFERENCES}

[1] P. Svestka, M. H. Overmars, M. H. Overmars, and M. H. Overmars, "Motion planning for car-like robots using a probabilistic learning approach," International Journal of Robotics Research, vol. 16, 1995.

[2] F. Lamiraux and J. P. Laumond, "Smooth motion planning for car-like vehicles," IEEE Transactions on Robotics and Automation, vol. 17, pp. 498-502, 2001.

[3] S. Thrun, M. Montemerlo, H. Dahlkamp, D. Stavens, A. Aron, J. Diebel, P. Fong, J. Gale, M. Halpenny, G. Hoffmann, K. Lau, C. Oakley, M. Palatucci, V. Pratt, P. Stang, S. Strohband, C. Dupont, L.-E. Jendrossek, C. Koelen, C. Markey, C. Rummel, J. van Niekerk, E. Jensen, P. Alessandrini, G. Bradski, B. Davies, S. Ettinger, A. Kaehler, A. Nefian, and P. Mahoney, "Stanley: The robot that won the DARPA Grand Challenge," Journal of Field Robotics, vol. 23, no. 9, pp. 661-692, 2006.

[4] A. Schindler, "Vehicle self-localization with high-precision digital maps," in Intelligent Vehicles Symposium (IV), 2013 IEEE, June 2013, pp. $141-146$.

[5] M. Hentschel and B. Wagner, "Autonomous robot navigation based on openstreetmap geodata," in Intelligent Transportation Systems (ITSC), 2010 13th International IEEE Conference on, Sept 2010, pp. 16451650.

[6] M. Buehler, K. Lagnemma, and S. Singh, "Special issue on the 2007 DARPA Urban Challenge, part I-III," in Journal of Field Robotics, M. Buehler, K. Lagnemma, and S. Singh, Eds., 2008, vol. 25, no. 8-10, pp. 423-860.

[7] J. Ziegler, P. Bender, M. Schreiber, H. Lategahn, T. Strauss, C. Stiller, T. Dang, U. Franke, N. Appenrodt, C. Keller, E. Kaus, R. Herrtwich, C. Rabe, D. Pfeiffer, F. Lindner, F. Stein, F. Erbs, M. Enzweiler, C. Knöppel, J. Hipp, M. Haueis, M. Trepte, C. Brenk, A. Tamke, M. Ghanaat, M. Braun, A. Joos, H. Fritz, H. Mock, M. Hein, and E. Zeeb, "Making bertha drive-an autonomous journey on a historic route," Intelligent Transportation Systems Magazine, IEEE, vol. 6, no. 2, pp. 8-20, Summer 2014.

[8] M. Haklay and P. Weber, "Openstreetmap: User-generated street maps," Pervasive Computing, IEEE, vol. 7, no. 4, pp. 12-18, Oct 2008.

[9] A. Victorino, P. Rives, and J.-J. Borrelly, "Safe navigation for indoor mobile robots,partii: Exploration, self-localization and map building," International Journal of Robotics Research, vol. 22, no. 12, pp. 10191041, 2003.

[10] E. C. M. Pereira, D. A. Lima, and A. C. Victorino, "Autonomous vehicle global navigation approach associating sensor based control and digital maps," in Robotics and Biomimetics, 2014. ROBIO '14. IEEE International Conference on, Dec 2014, pp. 2404-2409.

[11] O. Hassan, I. Adly, and K. Shehata, "Vehicle localization system based on ir-uwb for v2i applications," in Computer Engineering Systems (ICCES), 2013 8th International Conference on, Nov 2013, pp. 133137.

[12] D. Lima and A. Victorino, "A visual servoing approach for road lane following with obstacle avoidance," in Intelligent Transportation Systems (ITSC), 2014 IEEE 17th International Conference on, Oct 2014, pp. 412-417.

[13] J. Larsson, M. Broxvall, and A. Saffiotti, "Laser based intersection detection for reactive navigation in an underground mine," in Intelligent Robots and Systems, 2008. IROS 2008. IEEE/RSJ International Conference on, Sept 2008, pp. 2222-2227. 